\title{
Potentials and Limitations of Directional Deep Brain Stimulation: A Simulation Approach
}

\author{
Johanna Kramme ${ }^{a}$ Till A. Dembek ${ }^{b}$ Harald Treuer $^{a}$ Haidar S. Dafsari $^{b}$ \\ Michael T. Barbe ${ }^{b}$ Jochen Wirths ${ }^{a}$ Veerle Visser-Vandewalle ${ }^{a}$ \\ ${ }^{a}$ Department of Stereotactic and Functional Neurosurgery, University of Cologne Faculty of Medicine and \\ University Hospital Cologne, Cologne, Germany; ${ }^{b}$ Department of Neurology, University of Cologne Faculty of \\ Medicine and University Hospital Cologne, Cologne, Germany
}

\section{Keywords}

Directional deep brain stimulation · Lead-DBS · Volume of tissue activated $\cdot$ Lead orientation angle $\cdot$ Stereotactic accuracy

\begin{abstract}
Background: Directional leads are increasingly used in deep brain stimulation. They allow shaping the electrical field in the axial plane. These new possibilities increase the complexity of programming. Thus, optimized programming approaches are needed to assist clinical testing and to obtain full clinical benefit. Objectives: This simulation study investigates to what extent the electrical field can be shaped by directional steering to compensate for lead malposition. Method: Binary volumes of tissue activated (VTA) were simulated, by using a finite element method approach, for different amplitude distributions on the three directional electrodes. VTAs were shifted from 0 to $2 \mathrm{~mm}$ at different shift angles with respect to the lead orientation, to determine the best compensation of a target volume. Results: Malpositions of $1 \mathrm{~mm}$ can be compensated with the highest gain of overlap with directional leads. For larger shifts, an improvement of overlap of 10-
\end{abstract}

karger@karger.com www.karger.com/sfn

Karger $\stackrel{\text { ' }}{5}$

GOPEN ACCESS
(C) 2020 The Author(s)

Published by S. Karger AG, Basel

This is an Open Access article licensed under the Creative Commons Attribution-NonCommercial-4.0 International License (CC BY-NC) (http://www.karger.com/Services/OpenAccessLicense), applicable to the online version of the article only. Usage and distribution for commercial purposes requires written permission.
$30 \%$ is possible, depending on the stimulation amplitude and shift angle of the lead. Lead orientation and shift determine the amplitude distribution of the electrodes. Conclusion: To get full benefit from directional leads, both the shift angle as well as the shift to target volume are required to choose the correct amplitude distribution on the electrodes. Current directional leads have limitations when compensating malpositions $>1 \mathrm{~mm}$; however, they still outperform conventional leads in reducing overstimulation. Further, their main advantage probably lies in the reduction of side effects. Databases like the one from this simulation could serve for optimized lead programming algorithms in the future.

(C) 2020 The Author(s)

Published by S. Karger AG, Basel

\section{Introduction}

Using directional leads for deep brain stimulation (DBS) is the most recent approach in commercially available DBS devices. While with conventional, omnidirectional leads the electric field can only be adjusted along the lead axis, directional DBS also allows shaping the electrical field around the lead axis. Intraoperative studies 


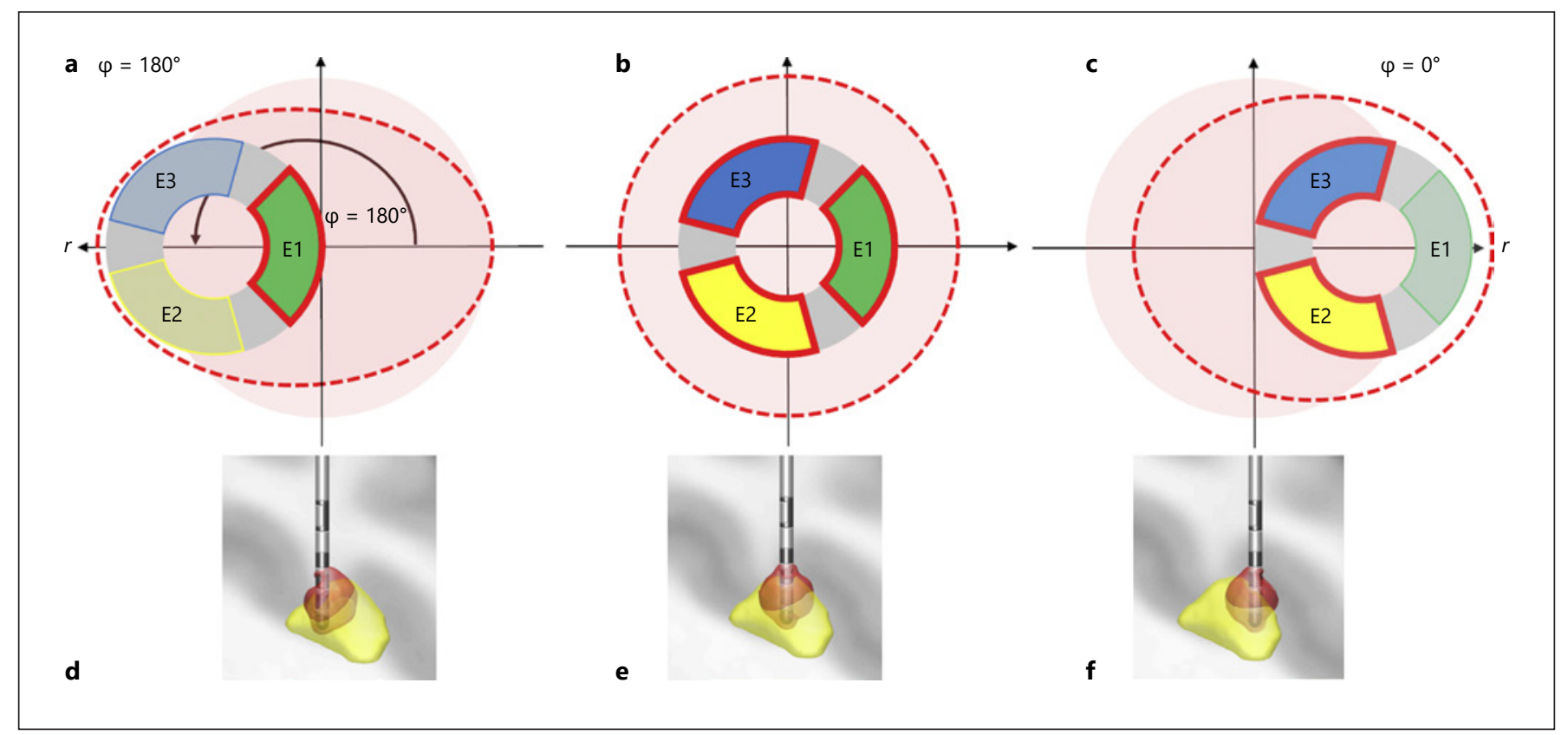

Fig. 1. a-c Schematic illustrations of a directional lead and VTA, in the axial plane. b, e Directional lead at target point in the STN, where the three electrodes are equally activated (omnidirectional stimulation). The red area schematically illustrates the size of the target volume and the dotted line the same VTA created by the three active contacts. a, $\mathbf{d}$ Malposition of $\mathrm{r}$ and shift angle $\varphi=180^{\circ}$. Only contact E1 is activated to compensate for the malposition.

with acute test stimulation $[1,2]$ as well as clinical studies with chronic DBS $[3,4]$ confirmed that this can help to control side effects and widen the therapeutic window. Still it is not clear whether long-term clinical outcome is also improved with directional compared to omnidirectional stimulation. Furthermore, directional lead programming is much more complex and time consuming for the clinician. There is a need for smart steering and field shaping $[5,6]$. Recent studies tried to identify sweet spots [7-9] within the subthalamic nucleus (STN) which could provide the basis for an optimized probabilistic directional lead programming approach. However, to develop such an approach it is equally important to know the technical limitations of directional stimulation. Li et al. [10] conclude in their review that the average targeting error when placing the lead is $1 \mathrm{~mm}$ with a standard deviation of $0.5 \mathrm{~mm}$. The aim of this simulation is to better understand to which extent directional steering may compensate in the case of malposition. Specifically, this study targets the potentials and limitations of directional stimulation in a simulation study, while focusing on two clinically relevant questions:
The dotted line represents the VTA created by E1. The plane red area is the target volume. $\mathbf{c}, \mathbf{f}$ Malposition of $\mathrm{r}$ and shift angle $\varphi=$ $0^{\circ}$. Electrode E2 and E3 are activated to compensate for the malposition. Their VTA is illustrated by the dotted line. $\mathbf{d}-\mathbf{f}$ Simulations of the three configurations in lead DBS, for $r=2 \mathrm{~mm}$ with an amplitude of $2 \mathrm{~mA}$, viewed from the sagittal plane. Red, VTA; yellow, STN.

1 From a surgical point of view, to what extent is it possible to compensate a malposition with directional stimulation?

2 From a neurological point of view, how much overstimulation can be avoided with directional stimulation when fully stimulating a target volume with a malpositioned lead?

\section{Materials and Methods}

To evaluate malposition compensation possibilities with directional DBS, omnidirectional target volumes were simulated for different amplitudes to serve as ground truth. Afterwards volumes of tissue activated (VTAs) for several directional settings of the electrodes E1, E2, and E3 in an omnidirectional lead were simulated. To investigate our study question, the lead was shifted by up to $2 \mathrm{~mm}$ from the target at different shift angles $(\varphi) . \varphi$ is defined as the angle (measured counterclockwise) between the horizontal axis through the middle of the lead in the axial plane and the axis at which the lead is shifted (compare with Fig. 2). In our first analysis the two extreme cases of shift angles in which the shift was either perfectly aligned with one directional electrode $\left(\varphi=180^{\circ}\right)$ or exactly between two directional electrodes $\left(\varphi=0^{\circ}\right)$ were investigated to determine 




Fig. 2. Directional electrodes at different shift angles with a shift of $r$. It can be seen that lead configurations are repeated every $120^{\circ}$. For a shift angle of $\varphi=30^{\circ}$, the amplitude distribution $(2 \mathrm{~mA})$ to the electrodes E1, E2, and E3 resulting in the best JC is shown for different shifts. For a shift of $2 \mathrm{~mm}$, the choice of amplitude distribution might be obvious but for small shifts a database is helpful for optimal programming approaches. the possibilities of compensating malposition depending on the distance from and the size of the target volume (Fig. 1). In our second analysis, different shift angles were investigated to determine the impact of lead orientation on compensation capabilities.

\section{Volume of Tissue Activated}

The finite element method approach [11] implemented in the Lead-DBS toolbox v2.1.8.1 (www.lead-dbs.org) [12] on a Linux machine was used to simulate binary VTAs with a finite element method approach [11]. VTAs were calculated for a Boston Scientific Cartesia Directional lead, which was placed in the STN of the distal atlas [13] and aligned with the $z$ axis of the coordinate system. The alignment of the segmented electrodes (E1, E2, E3) in the coordinate system is shown in Figure 1. An isotropic conductivity value of $\sigma=0.1 \mathrm{~S} / \mathrm{m}$ was chosen for the surrounding matter. The electric field threshold for binarization was set to $0.2 \mathrm{~V} / \mathrm{mm}$ which approximates the VTA radius estimate by Mädler and Coenen [11, $14,15]$. All VTAs were transferred to a standard space with isotropic $0.1 \mathrm{~mm}$ resolution.

\section{Target Volume}

As target volumes, VTAs were calculated with the lead on the target position and with all three directional electrodes activated with equal amplitudes (omnidirectional stimulation). These target volumes were calculated for amplitudes of $1-5 \mathrm{~mA}$ with a step size of $1 \mathrm{~mA}$.

\section{Shift Angle $\varphi$ of the Lead}

The lead was shifted along an axis $(r)$ at different shift angles $(\varphi)$ with respect to the lead orientation. As written earlier, $\varphi$ is defined as the angle (measured counterclockwise) between the horizontal axis through the middle of the lead in the axial plane and the axis at which the lead is shifted (Fig. 2). Seventeen shift angles were tested $\left(\varphi=0-120^{\circ}\right.$, step size $\left.7.5^{\circ}\right)$. The lead configurations were repeated every $120^{\circ}$, and results can be given for all angles $\left(0-360^{\circ}\right)$.

\section{VTA Simulation for Directional Settings}

With shifting the electrodes at different angles, different percentage distributions at the three electrodes of the omnidirectional ring (E1, E2, E3) were necessary to best compensate for a shift of the target position. This is due to the different orientations of the segmented electrodes at different shift angles, which is displayed in Figure 2. The combinations of percentage distributions, in total 81 combinations, were calculated as follows:

- All combinations must fulfill E1\% + E2\% + E3\% = 100\%

- For each electrode the percentage was changed by $10 \%$, permuting all possible combinations (66 combinations)

- Two electrodes have the same percentage with steps of 5\%, making combinations like $50-25-25$ possible (15 additional combinations)

The VTAs of the different percentage distributions were calculated as described above under VTA for 1-5 mA, in steps of $1 \mathrm{~mA}$. In the following the notation E1\%-E2\%-E3\% is used (e.g., 100$0-0=\mathrm{E} 1$ activated with $100 \%, 70-20-10=\mathrm{E} 1$ activated with $70 \%$, E2 with $20 \%$ and E3 with 10\%).

\section{Simulating Malposition and Calculating Overlap with Target} Volume

After generating the VTAs and bringing them to the standard space, all processing was done with Python 3.7 [16]. Within Py- 
Fig. 3. a For different amplitude distributions of E1, E2, and E3, the JC at different shifts to the ground truth is shown at $2 \mathrm{~mA}$. It is plotted for the two shift angles of $\varphi=$ $0^{\circ}$ and $\varphi=180^{\circ}$. The red line indicates the best possible JC adjusting the percentage distribution on the three electrodes according to the shift. The turquoise area represents the possible improvement of the JC in relation to omnidirectional stimulation and no shift. $\mathbf{b}$ The improvement of JC is plotted for different amplitude values and shifts from the target position. For smaller amplitudes, a higher gain is possible as for bigger amplitudes. The maximum improvement for $\varphi=180^{\circ}$ is reached at $1 \mathrm{~mm}$ and for $\varphi=0^{\circ}$ around $0.7 \mathrm{~mm}$.

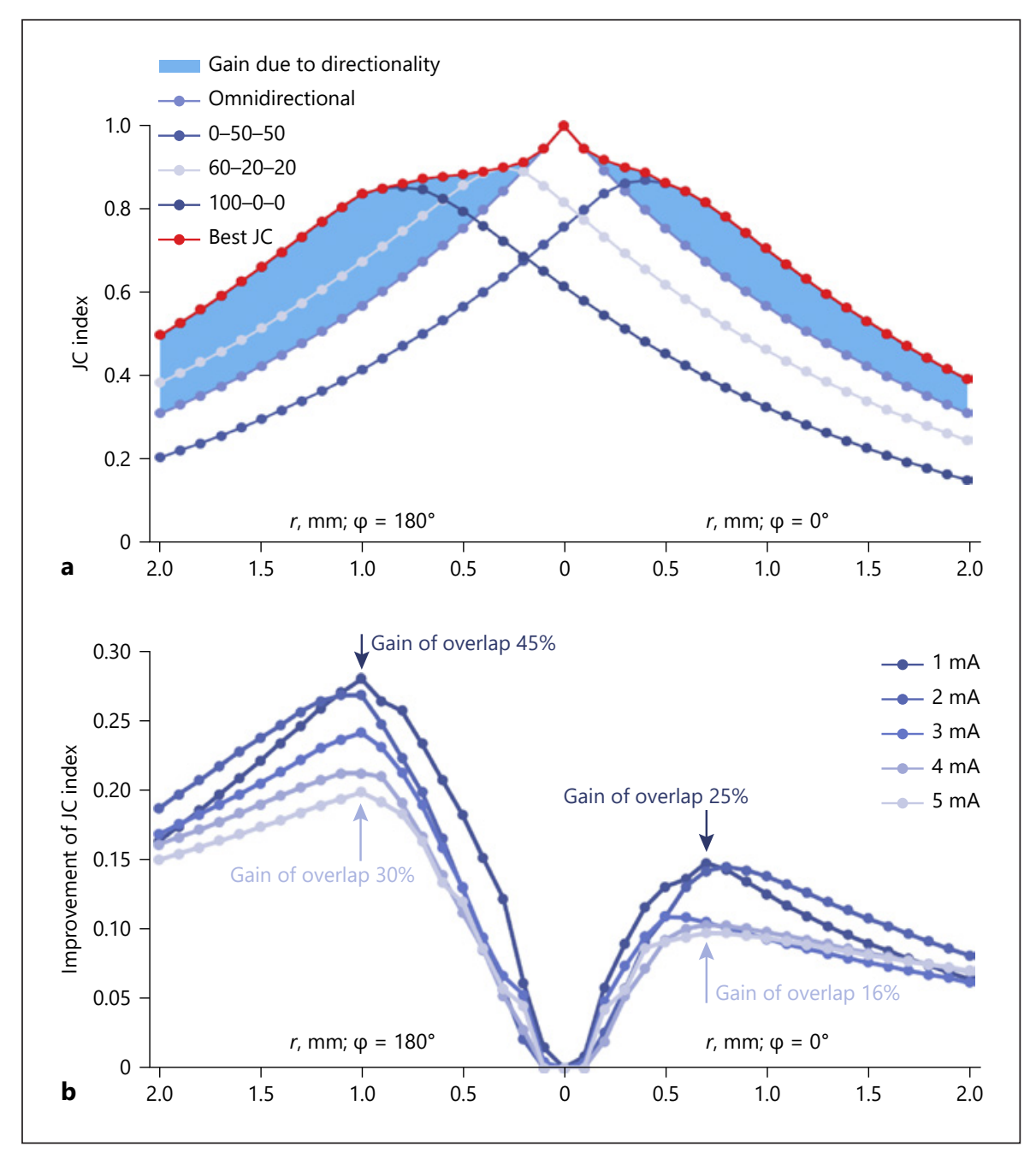

thon for representing a malposition the VTAs were shifted $r=0$ $2 \mathrm{~mm}$, step size of $0.1 \mathrm{~mm}$, at the different shift angles. The shifted VTAs were compared to the target volume with omnidirectional stimulation and with zero shift. To quantify the overlap of the VTAs the Jaccard index (JC) $[17,18]$ was used. It is defined as the size of the intersection divided by the size of the union of two sample sets.

The amount of volume of omnidirectional and directional stimulation is the same for equal amplitudes. Therefore, the best JC is achieved when the amplitude is kept the same and only the amplitude distribution of the three electrodes is varied to compensate for a malposition of the lead.

When target and stimulated volume have the same size, the JC can be converted to an overlap $([2 \mathrm{JC} /(1+\mathrm{JC})] \times 100)$, which signifies percentage of target volume overlapping with directional volume, since the overlap is a percentage value and might be a bit more intuitive. Due to this reason, it is additionally stated for some results. The gain of overlap is the subtraction of the JC at omnidirectional stimulation from the results of the JC at directional stimulation. The amount of gain of overlap depends on the shift angle and shift. The maximum shift for different amplitudes and shift angles is determined for an overlap of 70 and $90 \%$. The gain of overlap will be given as well.

The range of $r=0-2 \mathrm{~mm}$ was chosen due to the findings in the review of Li et al. [10], who report a targeting error of $1 \pm 0.5 \mathrm{~mm}$ and because the distance between the centers of two parallel trajectories used intraoperatively in microelectrode recording is usually $2 \mathrm{~mm}$. For each shift, the directional VTA with the best JC was identified, i.e. the stimulation settings with best compensation for the respective shift.

\section{Overstimulation with Full Target Coverage}

For shift angles of $\varphi=0^{\circ}$ and $\varphi=180^{\circ}$ with a shift of $r=0$ $2 \mathrm{~mm}$ (steps of $0.1 \mathrm{~mm}$ ), it was calculated how much the initial amplitude needs to be increased to fully overlap (99\%, $1 \%$ is statistical fluctuation in the simulation) the target volume. The calculation was done for VTAs with initial amplitudes of 1-4 mA, step size $1 \mathrm{~mA}$. If the amplitude to reach full coverage exceeded $5 \mathrm{~mA}$, the calculation was stopped, because data were only calculated for amplitudes up to $5 \mathrm{~mA}$. This is the reason why for target volumes of 3 and $4 \mathrm{~mA}$ the calculation was not possible for all shifts and no data are shown for $5 \mathrm{~mA}$, because full target coverage would need 


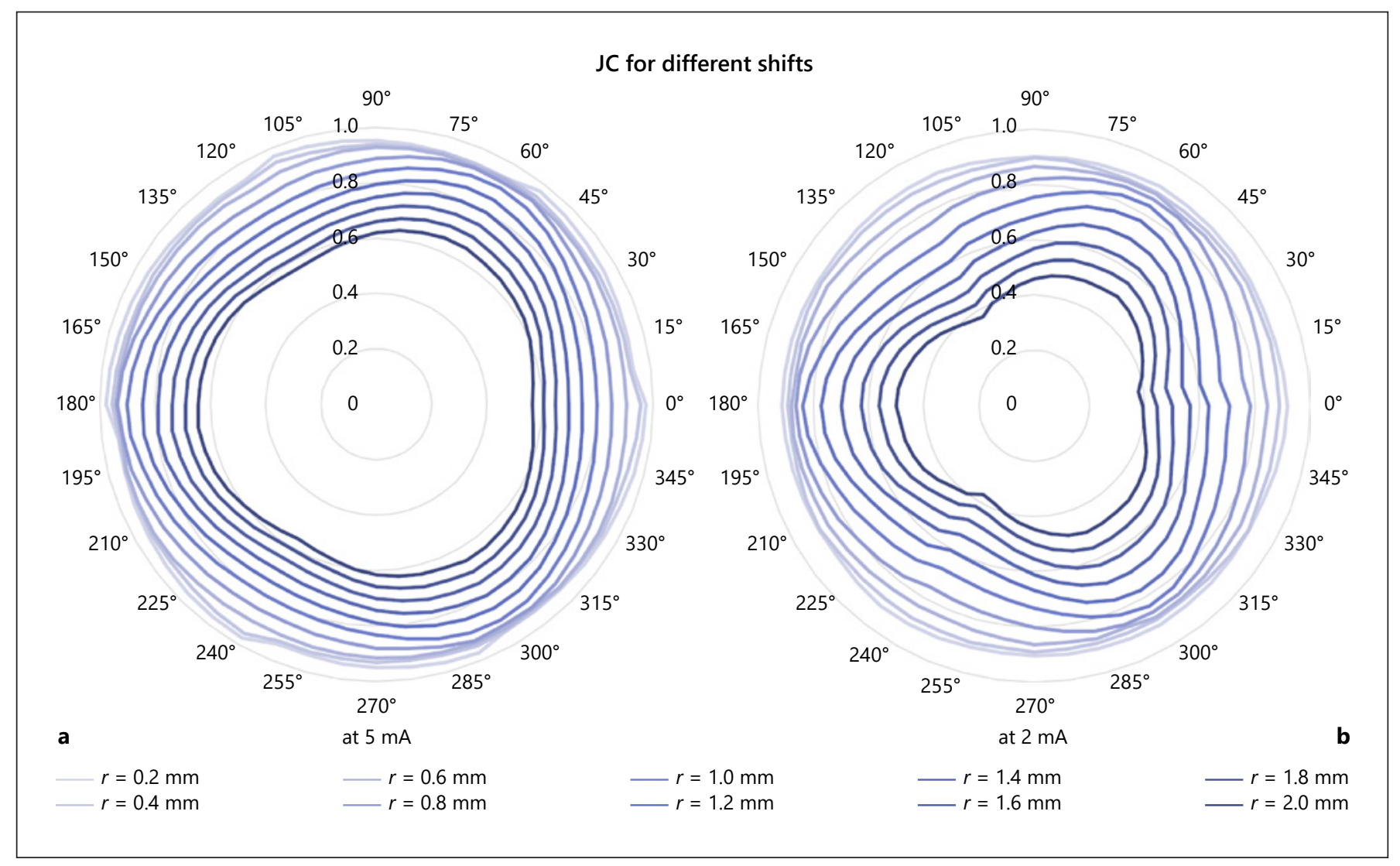

Fig. 4. Best JC at different shifts ( $r$ ), for each shift angle for $5 \mathrm{~mA}(\mathbf{a})$ and $2 \mathrm{~mA}(\mathbf{b})$. For smaller shifts, the JC is higher as well as for higher amplitudes.

amplitudes exceeding $5 \mathrm{~mA}$. The amount of stimulated out-oftarget volume in relation to the target volume was calculated. Optimized directionality, with best amplitude distribution to the three electrodes for a certain shift and shift angle was always regarded. For this analysis additional VTAs with an amplitude of 0.1-5.0 $\mathrm{mA}$, step size $0.1 \mathrm{~mA}$, were calculated.

\section{Results}

Directional Stimulation for $\varphi=0^{\circ}$ and $\varphi=180^{\circ}$

For every shift, another amplitude distribution to the three lead electrodes results in the best JC (Fig. 3).

When shifting the electrode so that the shift could be compensated by stimulation on only one electrode $(\varphi=$ $180^{\circ}$ ), the maximum JC improvement compared to omnidirectional DBS is reached at a shift of about $1 \mathrm{~mm}$. Here the gain of overlap lies between 30 and $45 \%$, being lowest for an amplitude of $5 \mathrm{~mA}$ and highest for an amplitude of $1 \mathrm{~mA}$. On the other hand, when the shift needed to be compensated by stimulation on two electrodes

Potentials and Limitations of Directional DBS with equal amplitude $\left(\varphi=0^{\circ}\right)$, the maximum benefit of directional stimulation is already reached at a shift of about $0.7 \mathrm{~mm}$. The gain of overlap is here $16-25 \%$ with respect to the different stimulation amplitudes (Fig. 3b).

It is possible to have an overlap of at least $70 \%$ (JC = 0.5 ) between best VTA for compensation and target volume for a shift of $2 \mathrm{~mm}$ for amplitudes of $2 \mathrm{~mA}$ and higher, and one electrode is needed for compensation. For $1 \mathrm{~mA}, 70 \%$ overlap is only possible for a maximum shift of $1.5 \mathrm{~mm}$. If two electrodes are needed for compensation, the maximum shift for $70 \%$ overlap is $1.2-2 \mathrm{~mm}$ depending on the stimulation amplitude. For an overlap of at least $90 \%(\mathrm{JC}=0.81)$ the maximum shift values drop to $0.7-1.3 \mathrm{~mm}$ for compensation with one electrode and to $0.5-1.0 \mathrm{~mm}$ for compensation with two electrodes. The results for all amplitudes are given in Table 1.

\section{Shift Angle of the Lead}

When investigating different shift angles, the highest JC values (Fig. 4) and most JC improvement compared to 
Table 1. For different amplitudes the maximum shift while having an overlap of $70 \%(J C=0.5)$ and $90 \%$ (JC $=0.81)$ between best VTA for compensation and target volume

\begin{tabular}{|c|c|c|c|c|c|c|c|c|}
\hline Amplitude & \multicolumn{4}{|c|}{ Lead shift angle $\varphi=0^{\circ}$} & \multicolumn{4}{|c|}{ Lead shift angle $\varphi=180^{\circ}$} \\
\hline $1 \mathrm{~mA}$ & $1.2 \mathrm{~mm}$ & $20 \%$ & $0.5 \mathrm{~mm}$ & $23 \%$ & $1.5 \mathrm{~mm}$ & $36 \%$ & $0.7 \mathrm{~mm}$ & $38 \%$ \\
\hline $2 \mathrm{~mA}$ & $1.6 \mathrm{~mm}$ & $19 \%$ & $0.7 \mathrm{~mm}$ & $25 \%$ & $2.0 \mathrm{~mm}$ & $31 \%$ & $1.1 \mathrm{~mm}$ & $42 \%$ \\
\hline $5 \mathrm{~mA}$ & $>2.0 \mathrm{~mm}$ & $13 \%$ & $1.0 \mathrm{~mm}$ & $17 \%$ & $>2.0 \mathrm{~mm}$ & $26 \%$ & $1.3 \mathrm{~mm}$ & $31 \%$ \\
\hline
\end{tabular}

The gain of overlap for this shift is also depicted.

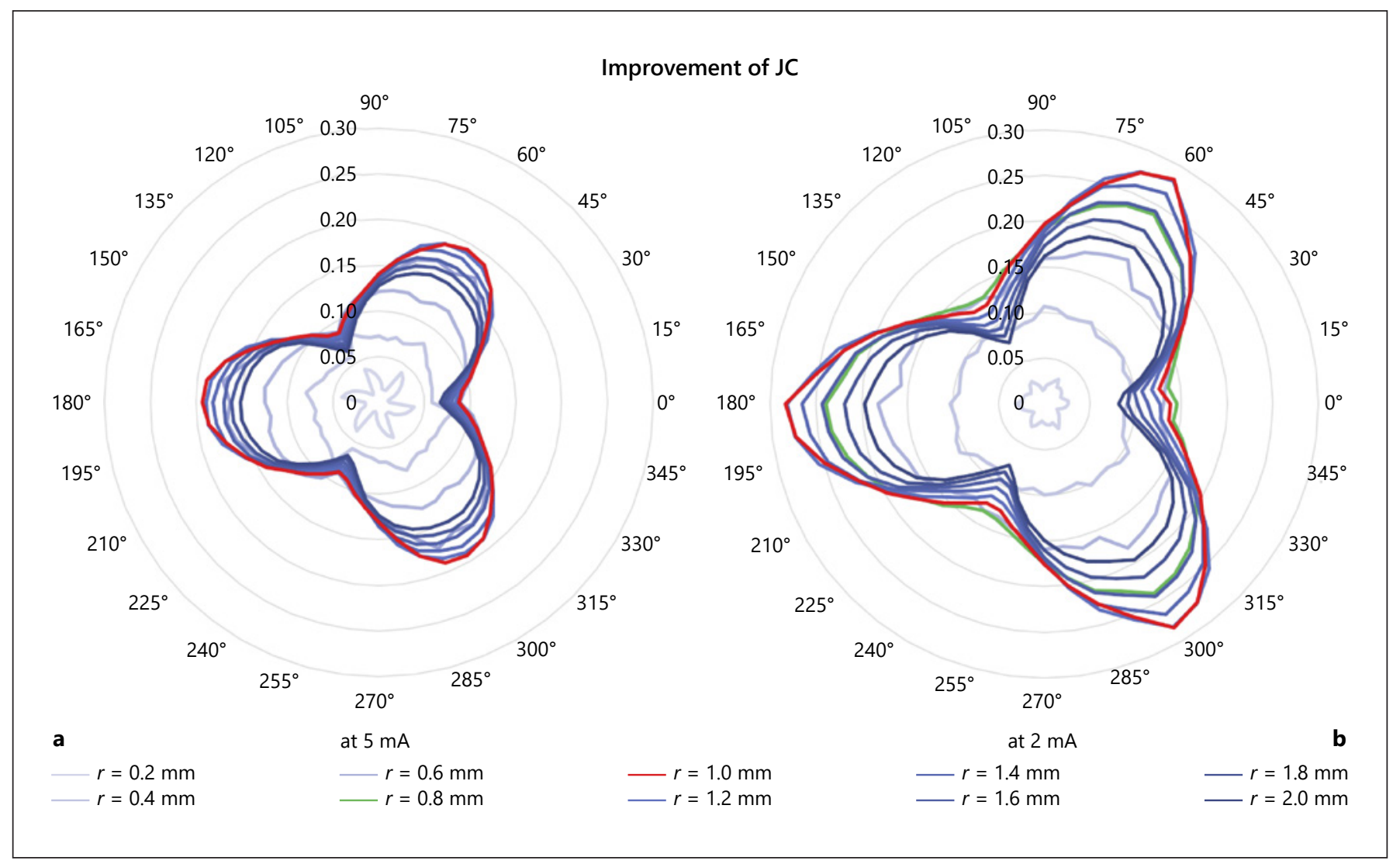

Fig. 5. Gain in JC considering shift and lead shift angle in relation to omnidirectional stimulation. This is equal to the blue area in Figure 3 but for all shift angles. The gain for higher amplitudes is less (a, $5 \mathrm{~mA}$ ) than for smaller amplitudes (b, $2 \mathrm{~mA}$ ). The best compensation is possible for $r=1.0 \mathrm{~mm}$ (red line) decreasing to $r=0.8 \mathrm{~mm}$ (green line) for smaller amplitudes with shift angles of 0,120 , and $240^{\circ}$.

omnidirectional stimulation (Fig. 5) are again seen for angles of 60,180 , and $300^{\circ}$, meaning angles in which the shift could be compensated via stimulation of only one segment. The lowest JC values on the other hand are seen for shift angles of 0,120 , and $240^{\circ}$, where the shift had to be compensated via equal stimulation on two segments. All other shift angles are configurations between these extremes. As before, with a higher amplitude higher JC 
Table 2. For different shift angles of the lead the maximum shift while having an overlap of $70 \%(J C=0.5)$ and $90 \%(J C=0.81)$ at amplitudes of 2 and $5 \mathrm{~mA}$

\begin{tabular}{|c|c|c|c|c|c|c|c|c|}
\hline \multirow[t]{2}{*}{ Shift angle } & \multicolumn{4}{|l|}{$2 \mathrm{~mA}$} & \multicolumn{4}{|l|}{$5 \mathrm{~mA}$} \\
\hline & $\begin{array}{l}\text { shift } \\
\mathrm{JC}>0.5 / \\
\text { overlap } 0.7\end{array}$ & $\begin{array}{l}\text { gain of } \\
\text { overlap }\end{array}$ & $\begin{array}{l}\text { shift } \\
\text { JC >0.81/ } \\
\text { overlap } 0.9\end{array}$ & $\begin{array}{l}\text { gain of } \\
\text { overlap }\end{array}$ & $\begin{array}{l}\text { shift } \\
\mathrm{JC}>0.5 / \\
\text { overlap } 0.7\end{array}$ & $\begin{array}{l}\text { gain of } \\
\text { overlap }\end{array}$ & $\begin{array}{l}\text { shift } \\
\mathrm{JC}>0.81 / \\
\text { overlap } 0.9\end{array}$ & $\begin{array}{l}\text { gain of } \\
\text { overlap }\end{array}$ \\
\hline $0^{\circ}$ & $1.6 \mathrm{~mm}$ & $19 \%$ & $0.7 \mathrm{~mm}$ & $25 \%$ & $2.3 \mathrm{~mm}$ & $11 \%$ & $1.0 \mathrm{~mm}$ & $17 \%$ \\
\hline $15^{\circ}$ & $1.6 \mathrm{~mm}$ & $25 \%$ & $0.7 \mathrm{~mm}$ & $36 \%$ & $2.4 \mathrm{~mm}$ & $15 \%$ & $1.1 \mathrm{~mm}$ & $19 \%$ \\
\hline $30^{\circ}$ & $1.8 \mathrm{~mm}$ & $28 \%$ & $0.8 \mathrm{~mm}$ & $30 \%$ & $2.6 \mathrm{~mm}$ & $20 \%$ & $1.2 \mathrm{~mm}$ & $24 \%$ \\
\hline $45^{\circ}$ & $1.9 \mathrm{~mm}$ & $36 \%$ & $0.9 \mathrm{~mm}$ & $25 \%$ & $2.6 \mathrm{~mm}$ & $21 \%$ & $1.3 \mathrm{~mm}$ & $29 \%$ \\
\hline $60^{\circ}$ & $2.0 \mathrm{~mm}$ & $31 \%$ & $1.1 \mathrm{~mm}$ & $37 \%$ & $2.7 \mathrm{~mm}$ & $21 \%$ & $1.4 \mathrm{~mm}$ & $31 \%$ \\
\hline $75^{\circ}$ & $1.9 \mathrm{~mm}$ & $33 \%$ & $1.0 \mathrm{~mm}$ & $40 \%$ & $2.6 \mathrm{~mm}$ & $21 \%$ & $1.3 \mathrm{~mm}$ & $30 \%$ \\
\hline $90^{\circ}$ & $1.8 \mathrm{~mm}$ & $29 \%$ & $0.8 \mathrm{~mm}$ & $32 \%$ & $2.6 \mathrm{~mm}$ & $20 \%$ & $1.2 \mathrm{~mm}$ & $25 \%$ \\
\hline $105^{\circ}$ & $1.7 \mathrm{~mm}$ & $22 \%$ & $0.7 \mathrm{~mm}$ & $26 \%$ & $2.4 \mathrm{~mm}$ & $16 \%$ & $1.0 \mathrm{~mm}$ & $20 \%$ \\
\hline $120^{\circ}$ & $1.5 \mathrm{~mm}$ & $18 \%$ & $0.7 \mathrm{~mm}$ & $24 \%$ & $2.3 \mathrm{~mm}$ & $11 \%$ & $1.0 \mathrm{~mm}$ & $16 \%$ \\
\hline
\end{tabular}

The gain of overlap for this shift is also depicted. Values for $5 \mathrm{~mA}$ and $70 \%$ overlap $(\mathrm{JC}=0.5)$ were extrapolated from the data of JC for shifts of $1.7-2.0 \mathrm{~mm}$ at the respective shift angle.

values can be reached as for the same shift with a smaller amplitude. For a shift of $r=2 \mathrm{~mm}$ the JC at $5 \mathrm{~mA}$ is between 0.55 and 0.65 but only between 0.4 and 0.5 at $2 \mathrm{~mA}$. If the gain in JC is compared, higher values are possible for smaller stimulation amplitudes. The maximum gain is for all shift angles at shifts between 0.8 and $1.0 \mathrm{~mm}$ (Fig. 5).

Table 2 summarizes the maximum shift for an overlap of $70 \%(\mathrm{JC}=0.5)$ and $90 \%(\mathrm{JC}=0.81)$ for rotation angles from 0 to $120^{\circ}$ at 2 and $5 \mathrm{~mA}$. The lowest shift values are for shift angles of 0 and $120^{\circ}$, the highest for $60^{\circ}$. For $5 \mathrm{~mA}$ $70 \%$ overlap can be reached for all shift angles, even exceeding shifts of $2 \mathrm{~mm}$. In Table 2 the maximum shift values are extrapolated for this case.

\section{Overstimulation with Full Target Coverage}

As can be seen in Figure 6, the needed increase in amplitude to fully stimulate the target volume for different shifts is almost independent from the initial amplitude and therefore the size of the target volume.

The amplitude for a full compensation increases rapidly and follows almost a linear trend. For the compensation with two electrodes the amplitude needs to be increased more than when compensating with one electrode. At $1 \mathrm{~mm}$ shift an original VTA of $2 \mathrm{~mA}$ needs already over $3 \mathrm{~mA}$ of final stimulation amplitude, and a 3-mA original VTA already exceeds the 4-mA final stimulation amplitude (Fig. 6a).

Increasing the stimulation amplitude to better cover a target volume comes with the disadvantage of also stimu- lating more out-of-target volume (Fig. 6b). The stimulated out-of-target volume in relation to the size of the target VTA was calculated. The smaller the target VTA, the more severe is the effect of stimulated out-of-target volume. For a VTA of $2 \mathrm{~mA}$ at $r=2 \mathrm{~mm}$ the stimulated out-of-target volume is more than double (200\%) the size of the target VTA. For a target VTA of $1 \mathrm{~mA}$ the stimulated out-of-target volume is between 4.5 and 6 times (450-600\%) the size of the target VTA depending on the shift angle.

\section{Discussion/Conclusion}

In this simulation study, we investigated to what extend the electrical field can be shaped using directional leads. Shaping of the field is important to compensate lead malposition and to avoid stimulation-induced side effects due to overstimulation. Programming approaches become very complex, due to the high degrees of freedom like: amplitude, frequency, pulse width, pulse shape, monopolar, bipolar stimulation, shift of the lead to target position, shift angle, directional or omnidirectional stimulation. Our paper shows the importance of knowledge of the shift to the target volume and the shift angle. The necessity to have databases for optimized programming approaches as well as the constraints in compensation of lead malposition are demonstrated.

The amount of volume of omnidirectional and directional stimulation is the same for equal amplitudes. 
Fig. 6. a Amount of amplitude increase for different initial amplitudes until the target volume is fully compensated. As a rule of thumb for shift angles of $\varphi=0^{\circ}$, the amplitude needs to be increased by $1.2 \mathrm{~mA}$ for a shift of $1 \mathrm{~mm}$ and by $1 \mathrm{~mA}$ per $1 \mathrm{~mm}$ shift for $\varphi=180^{\circ}$. The values are not calculated if the total amplitude exceeded $5 \mathrm{~mA}$. $\mathbf{b}$ The amount of stimulated out-of-target volume when the target volume is fully compensated. The smaller the target volume, the bigger is the stimulated out-of-target volume at full compensation. Optimized directionality, as best amplitude distribution depending on the shift and shift angle is regarded in both graphs.

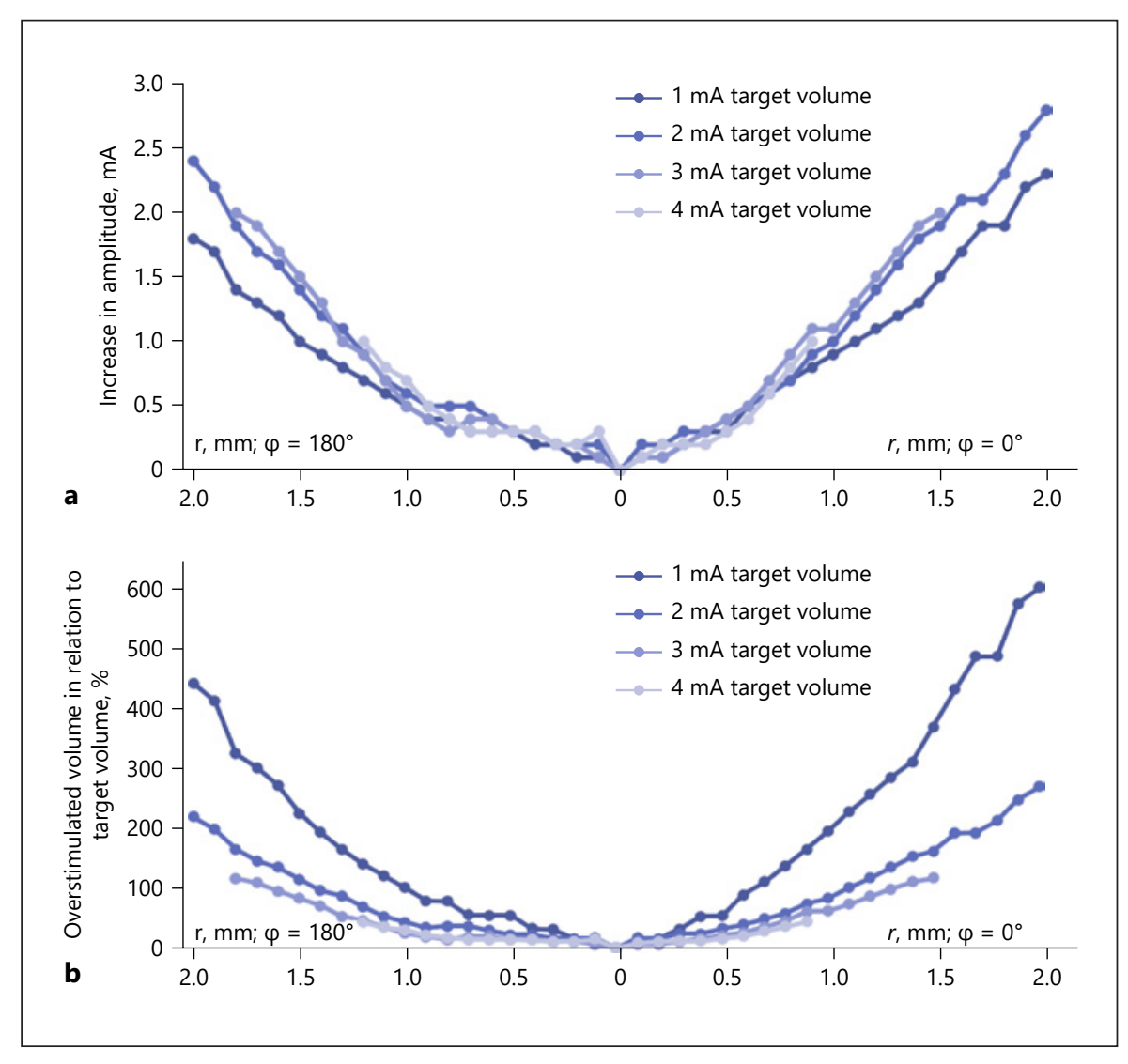

Therefore, the best JC is achieved when the amplitude is kept the same and only the amplitude distribution of the three electrodes is varied to compensate for a malposition of the lead. VTA volumes increase with rising amplitude. Bigger volumes can be compensated for with a higher JC. The reason is that, if a small volume is shifted the same distance as a bigger volume, the relative change is bigger for the small volume.

Overall, the results show that a malposition of the lead of $1 \mathrm{~mm}$ can be compensated for with the highest gain with directional stimulation. This might be less than expected, but also for greater malpositions an improvement in JC between 10 and $30 \%$ is possible. The gain due to directional stimulation should not be confounded with the total overlap of target area and stimulated volume. The overlap can be $70 \%$ for an amplitudes of $2 \mathrm{~mA}$ and above even for shifts of $2 \mathrm{~mm}$ with optimal lead rotation (compare Tables 1 and 2). Due to the limited range of compensation, the main advantage of the directional electrodes might be the reduction of side effects by steering the electric field away from critical areas. This is as well postulated by Anderson et al. [19], who nicely show the advantage of directional electrodes over circular ones in an op- timized programming approach that tries to maximize the target area by minimizing avoidance regions.

The knowledge of the shift angle is highly important as shown in Figures 4 and 5. Being unaware of the shift angle will lead to inefficient programming. All simulations were done with the same lead orientation, therefore the lead shift angles can easily be transferred to situations of lead rotation. Different groups [20-23] showed that it is possible to determine the rotation very precisely, and this knowledge should be used in optimized programming approaches. Especially, since deviations from the planned orientation of more than $30^{\circ}$ in $42 \%$ and of more than $60^{\circ}$ in about $11 \%$ of the leads were seen [24].

As shown in Figure 2, the optimal distribution of the amplitude distribution related to the three electrodes strongly depends on the shift and the shift angle. Therefore, it is proposed to establish databases to guide the programming. Due to statistic fluctuations in the simulation ( $1 \%$ volume change in repeated simulations), amplitude distributions might vary by $10-15 \%$ on the different electrodes but the main stimulation direction is kept the same.

Increasing the initial amplitude to compensate more of the target volume may easily result in side effects [19], 
since the amount of stimulated out-of-target volume increases rapidly especially for smaller target VTAs. As a rule of thumb for shift angles of $\varphi=0^{\circ}$, the amplitude needs to be increased by $1.2 \mathrm{~mA}$ to compensate a shift of $1 \mathrm{~mm}$ and by $1 \mathrm{~mA}$ per $1-\mathrm{mm}$ shift to compensate for $\varphi=$ $180^{\circ}$. All other shift angles have values between these two.

The study is limited by the fact that it only targets a shift in axial direction and no combination of segments along the $z$ axis is regarded. Moreover, we limited the model to equal values for gray and white matter resulting in an additional simplification [25]. Importantly, we also limited our analysis to monopolar stimulation settings. There is some evidence that combining directional and bipolar settings might lead to a further increase in side effect thresholds by avoiding stimulation spread in unwanted directions $[26,27]$. On the other hand, recent modeling studies suggest that the clinical effects of bipolar stimulation might be related to differential selectivity for stimulating axons running in different directions, and thus the classical VTA concept is insufficient to address the complexity of bipolar stimulation settings $[28,29]$. Despite these limitations, it becomes clear that a database to guide optimized programming approaches is necessary to fully benefit from the use of directional leads. More advanced models taking into account different tissue types, neuronal fibers, or other electric parameters will probably improve programming strategies, especially with regard to the prediction of side effects. However, they are not expected to change the general range of compensation with direction leads demonstrated in this study.

\section{Statement of Ethics}

Computer simulation study, no ethics apply.

\section{Conflict of Interest Statement}

Veerle Visser-Vandewalle reports advisory board membership payments and speaker honoraria, and grants from Boston Scientific and Aleva. Michael T. Barbe received speaker's honoraria from Medtronic, Boston Scientific, Abbott (formerly St. Jude), GE Medical, UCB, Apothekerverband Köln e.V., and Bial as well as research funding from the Felgenhauer-Stiftung, Forschungspool Klinische Studien (University of Cologne), Horizon 2020 (Gondola), Medtronic (ODIS), and Boston Scientific and advisory honoraria for the IQWIG. Till A. Dembek received speaker honoraria from Medtronic and Boston Scientific. Haidar S. Dafsari received honoraria from Medtronic and Boston Scientific. Jochen Wirths received speaker honoraria from Boston Scientific. All other authors report no disclosures.

\section{Funding Sources}

Granted by EIT-Health. Project 19109: DBS-Select-Selectiv Deep Brain Stimulation: Time and space for a change?

\section{Author Contributions}

Study design: all authors. Computer simulation: Kramme, Dembek. Data evaluation: all authors. Drafting the work: Kramme. Final approval: all authors.

\section{References}

1 Pollo C, Kaelin-Lang A, Oertel MF, Stieglitz L, Taub E, Fuhr P, et al. Directional deep brain stimulation: an intraoperative double-blind pilot study. Brain. 2014 Jul;137(Pt 7):201526.

2 Contarino MF, Bour LJ, Verhagen R, Lourens MA, de Bie RM, van den Munckhof $P$, et al. Directional steering: A novel approach to deep brain stimulation. Neurology. 2014 Sep; 83(13):1163-9.

3 Dembek TA, Reker P, Visser-Vandewalle V, Wirths J, Treuer H, Klehr M, et al. Directional DBS increases side-effect thresholds-A prospective, double-blind trial. Mov Disord. 2017 Oct;32(10):1380-8.

4 Steigerwald F, Matthies C, Volkmann J. Directional Deep Brain Stimulation. Neurotherapeutics. 2019 Jan;16(1):100-4.

5 Deeb W, Giordano JJ, Rossi PJ, Mogilner AY, Gunduz A, Judy JW, et al. Proceedings of the Fourth Annual Deep Brain Stimulation Think
Tank: a review of emerging issues and technologies. Front Integr Neurosci. 2016;10:38.

6 Hariz M. Deep brain stimulation: new techniques. Parkinsonism Relat Disord. 2014 Jan; 20 Suppl 1:S192-6.

7 Dembek TA, Roediger J, Horn A, Reker P, Oehrn C, Dafsari HS, et al. Probabilistic sweetspots predict motor outcome for deep brain stimulation in Parkinson's disease. Ann Neurol. 2019 Oct;86(4):527-38.

8 Nguyen TA, Djilas M, Nowacki A, Mercanzini A, Schüpbach M, Renaud P, et al. Analysis of patient-specific stimulation with segmented leads in the subthalamic nucleus. PLoS One. 2019 Jun;14(6):e0217985.

9 Petry-Schmelzer JN, Krause M, Dembek TA, Horn A, Evans J, Ashkan K, et al.; EUROPAR and the IPMDS Non-Motor PD Study Group. Non-motor outcomes depend on location of neurostimulation in Parkinson's disease. Brain. 2019 Nov;142(11):3592-604.
10 Li Z, Zhang JG, Ye Y, Li X. Review on Factors Affecting Targeting Accuracy of Deep Brain Stimulation Electrode Implantation between 2001 and 2015. Stereotact Funct Neurosurg. 2016;94(6):351-62.

11 Horn A, Reich M, Vorwerk J, Li N, Wenzel G, Fang Q, et al. Connectivity Predicts deep brain stimulation outcome in Parkinson disease. Ann Neurol. 2017 Jul;82(1):67-78.

12 Horn A, Li N, Dembek TA, Kappel A, Boulay C, Ewert S, et al. Lead-DBS v2: towards a comprehensive pipeline for deep brain stimulation imaging. Neuroimage. 2019 Jan; 184: 293-316.

13 Ewert S, Plettig P, Li N, Chakravarty MM, Collins DL, Herrington TM, et al. Toward defining deep brain stimulation targets in MNI space: a subcortical atlas based on multimodal MRI, histology and structural connectivity. Neuroimage. 2018 Apr; 170: 271-82. 
14 Mädler B, Coenen VA. Explaining clinical effects of deep brain stimulation through simplified target-specific modeling of the volume of activated tissue. AJNR Am J Neuroradiol. 2012 Jun;33(6):1072-80.

15 Astrom M, Diczfalusy E, Martens H, Wardell K. Relationship between neural activation and electric field distribution during deep brain stimulation. IEEE Trans Biomed Eng. 2015 Feb;62(2):664-72.

16 Python (cited 2019 Aug 7). Available from: https://www.python.org/

17 Jaccard P. The distribution of the flora in the Alpine Zone. New Phytol. 1912;11(2):37-50.

18 Shi R, Ngan KN, Li S. Jaccard index compensation for object segmentation evaluation. In: 2014 IEEE International Conference on Image Processing (ICIP); 10/27/2014 10/30/2014; Paris, France. Piscataway, NJ: IEEE; 2014. p. 4457-61.

19 Anderson DN, Osting B, Vorwerk J, Dorval $\mathrm{AD}$, Butson CR. Optimized programming algorithm for cylindrical and directional deep brain stimulation electrodes. J Neural Eng. 2018 Apr;15(2):026005.
20 Hellerbach A, Dembek TA, Hoevels M, Holz JA, Gierich A, Luyken K, et al. DiODe: Directional Orientation Detection of Segmented Deep Brain Stimulation Leads: A Sequential Algorithm Based on CT Imaging. Stereotact Funct Neurosurg. 2018;96(5):335-41.

21 Hunsche S, Neudorfer C, Majdoub FE, Maarouf M, Sauner D. Determining the Rotational Orientation of Directional Deep Brain Stimulation Leads Employing Flat-Panel Computed Tomography. Oper Neurosurg (Hagerstown). 2019 Apr;16(4):465-70.

22 Reinacher PC, Krüger MT, Coenen VA, Shah M, Roelz R, Jenkner C, et al. Determining the Orientation of Directional Deep Brain Stimulation Electrodes Using 3D Rotational Fluoroscopy. AJNR Am J Neuroradiol. 2017 Jun; 38(6):1111-6.

23 Sitz A, Hoevels M, Hellerbach A, Gierich A, Luyken K, Dembek TA, et al. Determining the orientation angle of directional leads for deep brain stimulation using computed tomography and digital $\mathrm{x}$-ray imaging: A phantom study. Med Phys. 2017 Sep;44(9):4463-73.

24 Dembek TA, Hoevels M, Hellerbach A, Horn A, Petry-Schmelzer JN, Borggrefe J, et al. Directional DBS leads show large deviations from their intended implantation orientation. Parkinsonism Relat Disord. 2019 Oct:67:117-21.
25 Nordin T, Zsigmond P, Pujol S, Westin CF, Wårdell $\mathrm{K}$. White matter tracing combined with electric field simulation - a patient-specific approach for deep brain stimulation. Neuroimage Clin. 2019;24:102026.

26 Steffen JK, Reker P, Mennicken FK, Dembek TA, Dafsari HS, Fink GR, et al. Bipolar Directional Deep Brain Stimulation in Essential and Parkinsonian Tremor. Neuromodulation. 2020 Jun;23(4):543-9.

27 Soh D, Ten Brinke TR, Lozano AM, Fasano A. Therapeutic Window of Deep Brain Stimulation Using Cathodic Monopolar, Bipolar, Semi-Bipolar, and Anodic Stimulation. Neuromodulation. 2019 Jun;22(4):451-5.

28 Slopsema JP, Peña E, Patriat R, Lehto LJ, Gröhn O, Mangia S, et al. Clinical deep brain stimulation strategies for orientation-selective pathway activation. J Neural Eng. 2018 Oct;15(5):056029.

29 Anderson DN, Duffley G, Vorwerk J, Dorval $\mathrm{AD}$, Butson CR. Anodic stimulation misunderstood: preferential activation of fiber orientations with anodic waveforms in deep brain stimulation. J Neural Eng. 2019 Feb; 16(1):016026. 